\title{
Remote Health Monitoring During and After the COVID-19 Pandemic
}

\author{
Nikita SHKLOVSKIY-KORDI ${ }^{\mathrm{a}, 1}$, Lev EVELSON ${ }^{\mathrm{b}}$, Irina KARGALSKAYA ${ }^{\mathrm{c}}$, Sergei \\ SHINKARIOV $^{\mathrm{d}}$, Olga KREMENETSKAYA ${ }^{\mathrm{e}}$ and Boris ZINGERMAN ${ }^{\mathrm{f}}$ \\ ${ }^{\mathrm{a}}$ National Research Center for Hematology, Russia \\ ${ }^{\mathrm{b}}$ Innovation Scientific Center of Remote Information Technologies, Russia \\ "The Committee "Patient-oriented Telemedicine", Russia \\ ${ }^{d}$ Lipetsk Oncological Hospital, Russia \\ ${ }^{\mathrm{e}}$ Center for Theoretical Problems of Physico-chemical Pharmacology, Russia \\ ${ }^{\mathrm{f}}$ TelePat LLC, Russia
}

\begin{abstract}
High importance of the remote health monitoring (RHM) especially during and after the pandemic is accentuated in this article. It is displayed that by this way it is possible to revealing automatically situations "suspicious" from the COVID-19 illness view point for some concrete patients and also to keep up with possible complications after the illness. Another great opportunity of the RHM is connected with investigation of the vaccines. RHM allows reveal cases of the complications among the patients as well as positive and negative consequences of the vaccination. The general depersonalized data should be gathered and analyzed to research the regularities of the COVID-19 sickness rate and the complications frequency and also of the safety and effectiveness of the vaccines. It is proposed to combine advantages of RHM for medical care for concrete patients with opened opportunities for research to reveal important general normality connected with correlation between COVID-19 complications and peculiarities of various chronic diseases and specific medical statements. Telemedicine platform intended for creation of RHM systems is described. It has gone through the pilot running in Russian medical institutions and now presented good results have been achieved.
\end{abstract}

Keywords. Remote health monitoring, pandemic, complications, vaccination

\section{Introduction}

Remote health monitoring (RHM) is important tool in the telemedicine segment "patient - physician". Numerous investigations displayed that remote health monitoring could improve medical care significantly [1]. COVID-19 pandemic has increased some more actuality of this way of medical care. RHM allows avoid face-to-face contacts between patient and physician and it is very important during the pandemic. In this article results of the pilot implementation of the telemedicine platform developed in Russia are presented. This platform had already been successfully used for remote health monitoring cancer patients and also several other groups. When COVID-19 pandemic

\footnotetext{
${ }^{1}$ Corresponding Author, Nikita Shklovskiy-Kordi, National Research Center for Hematology, 4 Noviy Zykovskiy pr., Moscow, 125167, Russia; E-mail: nikitashk@gmail.com
} 
started, the elements connected with new challenges had been added. Soon (and probably already now) problems connected with the pandemic consequences would be topical. We think that RHM is very good tool for solution of them. In this article these perspectives are discussed.

The investigation of the long-term consequences of the pandemic can touch many different problems. Some of them are connected with physiological processes in the human organism; other ones are corresponding to psychological stresses which can take place for all monitored patients.

Besides of the COVID-19 consequences, the RHM can help to research effectiveness and possible negative consequences of the vaccination.

\section{Description of used methods and the telemedicine platform}

The main method to research and solve the above problems is to take working telemedicine platform, adapt it according with the research task and to start monitoring. The special telemedicine platform "Medsenger" [2] had been developed earlier. It is intended for RHM. There are peculiarities which give significant advantages as compare with universal messengers (for example, WhatsApp) and other telemedicine products. The Medsenger provides all types of interaction between a patient and a doctor through a special channel established by the medical institution. So, the questions, answers, messages, transferred documents, and other information units are automatically recorded and can be monitored by the clinic. A doctor hasn't to give his personal contact data. There are various generators of questionnaires, automatic reminders, special visualization means presenting parameters of the patient health statement and other convenient tools. These means unload a doctor from routine work and allow estimate situation better. As compare with other telemedicine platforms "patient-physician" devoted to the remote health monitoring, the Medsenger is less oriented toward IoT and more toward connection with system EHR (electronic health records). There is a great scientific and practical medical experience which is used in various concrete solutions developed on the Medsenger platform. Generation of the questionnaires and processing of them after filling up, analysis of the data transferred from a patient and other actions are done according with the experience which had been structured and stored within the telemedicine platform.

RHM here is considered as continuation and element of general medical care realized by the attending doctor. The principal method to form interaction between patient and physician in the Messendger is asynchronous: a patient asks when he wants to ask and a physician answers when he has the opportunity, but necessarily during the period stipulated in the contract between the patient and the medical institution (working day, 12 hours, 24 hours, etc.). The subsystem of revelation of the alarms automatically analyses information transferred from a patient and notifies the attending doctor if the situation demanding his immediate attention. There are convenient means for visualization which allow estimate health statement of the patient. A doctor can see also a "picture" of all patients involved into RHM under his supervision. The example is presented in the Figure 1. There are graphs of systolic and diastolic blood pressure and pulse rate which took place in the RHM. All these graphs are displayed in the common time axis together with the markers reflecting receiving of drugs. 


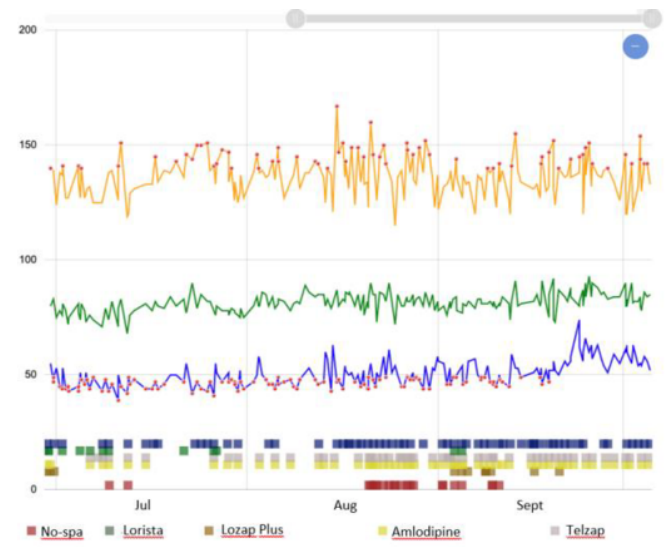

Figure 1. Example of the visualization tool

\section{A Pilot Utilization of the Telemedicine Platform}

\subsection{The main results}

Several RHM systems have already been developed on the basis of the Medsenger telemedicine platform: for cancer patients, for patients who had a kidney transplantation, for pregnant women, etc. A pilot operation of the telemedicine system ONCONET [3] developed for remote health monitoring cancer patients during rehabilitation after chemotherapy took place in 2018 - 2020 years. 22 medical institutions from 10 regions of Russia and 174 doctors participated in the testing of the system. The largest number of patients has been tested in the Lipetsk Oncological Hospital where 61 doctors and 206 cancer patients took part in the RHM project. The study involved patients with breast cancer, lung, cervical and ovarian cancer, prostate, thyroid, kidney, stomach and colorectal cancer, lymphoma. The reference group of patients not involved into remote monitoring (209 persons) had been selected. Similarity of diagnoses, stages of diseases, treatment regimens and the sex-age composition was observed. The feedback from doctors and patients was positive. The main quantitative results are presented in the Table 1 .

Table 1. Results of the pilot utilization of the ONCONET remote health monitoring system

\begin{tabular}{ccc}
\hline Complication type & $\begin{array}{c}\text { Part of patients under } \\
\text { monitoring with } \\
\text { complications, \% }\end{array}$ & $\begin{array}{c}\text { Part of patients with } \\
\text { complications in reference } \\
\text { group , \% }\end{array}$ \\
\hline Hematological & 32 & 42 \\
Gastroduodenal & 0,7 & 2,6 \\
Neurotoxic & 0 & 3,2 \\
Nephrotoxic & 3,6 & 13,6 \\
Skin & 0 & 1,6 \\
Postponements of the next & 7,9 & 11,9 \\
chemotherapy course & & \\
\hline
\end{tabular}

\subsection{Supplementation to the system during the COVID-19 Pandemic}

After that the COVID-19 pandemic had been started some elements were added to detect cases with a big probability of the COVID-19 illness [4]. In particular, questions 
regarding the COVID-19 qualitative symptoms together with analysis of objective quantitative parameters (for example, body temperature) allow reveal such situations. In those cases the urgent message is sent to the patient and to his attending doctor.

The ONCONET includes electronic library containing information connected with various types of cancer. There are 1,250 pages of patient-oriented information content and supporting patient teaching cases from leading experts on the specifics and treatments for life and nutrition, rehabilitation and care. It is structured on oncologic diagnoses (21 types of cancer) as well as stages of the patient's life. 13 video teaching cases have been added to the library of the system after the pandemic start (the teaching cases were done together with prof. N.V.Zhukov). They concern the life conditions of oncologic patients under the COVID-19 quarantine situation. Those cases include many important recommendations such as: how to control breath under the COVID-19, prophylaxis peculiarities for patients with cancer, psychological aspects during pandemic.

\section{Discussion about Perspectives for Research of the COVID-19 and the Vaccination Consequences}

RHM can be used for research and practical medical care to reveal and reduce negative consequences of the pandemic. According with the patient-oriented approach [5-7], decision making systems for patients and doctors have to take place. Patients must have an opportunity to know their own medical data and take part in decision making. The following tasks can be picked out: detecting of the COVID-19 complications in the health of concrete patient, revelation of general regularities connected with effectiveness and safety of the vaccines and with frequency of various COVID-19 complications. The first problem can be solved by the same methods and means which are already used in the Medsenger platform now. It should only mark cases - "COVID-19 illness earlier took place" or "not" in the data base. In order to reveal general regularities it's needed to turn to analysis of the depersonalized data. Such data as age, sex, presence of COVID-19 disease in the health history, fact (including the type of the vaccine), date of vaccination and medical data should be considered. After consolidation of the depersonalized data the analysis should be done by statistical methods. Effectiveness of the vaccine can be checked in comparison of the frequency of COVID-19 hard illness after vaccination with some established limit value or with the frequency in the reference group. The vaccine safety can be estimated by determination of the statistical significance of the difference between frequencies of negative health changes after vaccination and without vaccination. The investigation of difference between results of vaccination by various vaccines can be done analogously. Various types of complications taking place after the COVID-19 illness should be also researched. Such analysis should be done periodically within permanent monitoring to reveal the long-term tendencies. If the scale of the RHM would become very large, the Big Data, AI and Data Mining methods should be also used for analysis of collected quantitative and qualitative depersonalized medical data. Analogous approach can be used for various RHM systems developed on a foundation of the Medsenger. It would provide improvement of medical care for different categories of patients and also the opportunity to detect important regularities of correlation between the problems of the main type of the patient medical position (statement after transplantation, pregnancy, chronic illness, etc.) and COVID-19 consequences.

Another important problem which should be solved by RHM is connected with psychological consequences of the pandemic. They would be detected by analysis of the 
questionnaires developed by psychologist (or by the attending doctor according with the recommendations of a psychologist) and filled up by the patients.

\section{Conclusion}

RHM is a very useful way of medical care during and after the COVID-19 pandemic. It should be also used to research the negative consequences of the COVID-19 (including psychological sequels) and to investigate effectiveness and safety of various vaccines against the COVID-19.

The software system ONCONET developed on a base of the telemedicine platform Medsenger gave good results for monitoring of the cancer patient's health. The system had been supplemented taking into account the COVID-19 aspects. For near perspective it should include the means of analysis of the depersonalized data to reveal important general regularities and to solve the problems connected with research of the COVID-19 consequences and of effectiveness and safety of the vaccines. The same way should be realized for various categories of patients in other systems developed on a base of the Medsenger. It should provide improvement of the medical care and investigation of some general regularity of a combination of the COVID-19 consequences and peculiarities of the patient's classes.

\section{Acknowledgments}

This work was supported in part by the Ministry of Science and Higher Education of the Russian Federation (AAAA-A18-118012390247-0) and by RFBR grant (project № 19-07-01235).

\section{References}

[1] Topol E. Digital medicine: empowering both patients and clinicians. The Lancet. 2016 Aug; 388(10046): 740-1.

[2] Shklovskiy-Kordi N, Borodin R, Zingerman B, Shifrin M, Kremenetskaya O, Vorobiev A. Web-Service Medical Messenger - Intelligent Algorithm of Remote Counseling. HIS 2018;LNCS(11148):1-5

[3] Kargalskaja I, Shinkarev S, Zingerman B, Fistul I, Nozik A, Arseniev S. ONCONET - a digital service for remote monitoring and support of cancer patients on chemotherapy. Proceedings of the IADIS International Conference e-Health; 2020 Jul 21-23;Virtual Conference: IADIS Press; 2020. p. 193-8..

[4] Shinkariov S, Zingerman B, Kargalskaya I, Nozik A, Fistul I, Evelson L, Kremenetskaya A, Sun Le, Jun $\mathrm{Xu}$, Kremenetskaya O, Shklovskiy-Kordi N. Telemedicine System with Elements of Artificial Intelligence for Health Monitoring during COVID-19 Pandemic. HIS 2020;LNCS(12435):103-10.

[5] Peleg M, Shahar Y, Quaglini S., Fux A, Garcia-Sanchez G, Goldstein A, Hernando MH, Klimov D, Martinez-Sarriegui I, Napolitano C, Rigla M, Sacchi L, Shalom E. Soffer P. MobiGuide: a personalized and patient-centric decision-support system and its evaluation in the atrial fibrillation and gestational diabetes domains. User Modeling and User Adapted Interaction 2017;27(2):159-213.

[6] Morgan AU, Balachandran M, Do D, Lam D, Parambath A, Chaiyachati KH, Bonalumi NM, Day SC, Lee KC, Asch DA. Remote Monitoring of Patients with Covid-19: Design, implementation, and outcomes of the first 3,000 patients in COVID Watch. NEJM Catal Innov Care Deliv.2020 Jul;1(4):1-12.

[7] Bailey C, Black JRM, Swanton C. Cancer Research: The Lessons to Learn from COVID-19. Cancer Discov. 2020 Sep;10(9):1263-1266. 